\section{Access to drugs on clinical trial}

SIR-I was amused by Dr Vincent T. DeVita's comments reported in your article on Biotherapeutics Inc. (Nature 325, 99; 1987). Many patients who have attempted to take part in National Cancer Institute (NCI) trials would dispute that these new treatments are being made "available to the public as fast as is prudent". Clearly, there are many new approaches available for the treatment of cancer and these are not being made quickly available. There are many patients who wish to try experimental treatment who are unable to gain access to such approaches because of the restrictive nature of the regulated monopoly administered by the government/ academic sector. Nature should poll patients with respect to DeVita's claim.

It is puzzling to continue to see the word ethics coming from the NCI. Patients do fund research at Biotherapeutics but the public should know that patients also pay for experimental approaches in the NCI/ university system. When I was director of the Biological Response Modifiers Program for DeVita, we billed insurance companies for the cost of hospital care at Frederick Memorial Hospital while patients received experimental treatment with interferon and other new biological approaches. Thus, the patients, through their insurance company, clearly "paid for experimental treatment" in that NCI study. In addition, it is well known that university researchers allow and encourage their hospitals to bill insurance companies and sometimes patients for the cost of hospitalization when patients are on $\mathrm{NCI} /$ university experimental protocols. Many of these trials are initiated and monitored by the NCI and experimental drugs made available for these trials through the NCI. Thus, patients and their insurance companies are paying for the clinical costs in these experimental trials. According to DeVita's standard, is not that unethical?

Robert K. Oldham Biological Therapy Institute,

Riverside Drive,

Franklin, Tennessee 37064, USA

\section{Problems facing university science}

SIR-The recent pay awards agreed by the Association of University Teachers favour those at the top end of the scientific profession and will not help in attracting the younger researchers on whom the future of science in Britain ultimately rests. To do a $\mathrm{PhD}$ to enter university research in Britain these days is to condemn oneself to years of penury and constant insecurity. When senior scientists next request more funding for basic research (and basic jobs), the government's response will be that money was made available and that it went straight into the pockets of the supplicants, already overpaid in the prime minister's eyes, assuming that her recent statements about cutting top rates of income tax to stem the brain drain are to be taken at face value. The voting public will offer science at the universities little sympathy, even if we bend over backwards to show how useful and applied, cheap and streamlined we have become.

We will not prevent the erosion of British university science by permitting the award of such selective pay increases and by aiding and abetting the government in its plans for rationalization of research and teaching. It is little use pretending that we can be 'great value for money' in the short term. We cannot be trained to turn basic research into pounds sterling within the life of a government or two. This particular government, concerned as it is with value for money and the quick turnover of the marketplace, knows that basic research is expensive. It also knows that financial returns on the investment can be enormous but often take decades to appear. The pursuit of basic research, the task for which the universities are best equipped, is thus anathematic to Thatcherism and the present government is relatively unconcerned when we leave to ply our expensive trade abroad. Britain's loss is deferred to the 'never-never'. We must remind the electorate and political parties of the necessity of investment in university research, despite its long-term objectives, refuse to assist in the removal of the basic research and teaching roles of the universities and, most importantly, ensure that the little money cast in our direction is not spent on pay rises which merely allow those remaining in British science to watch impotently over its demise in slightly greater comfort.

\section{Department of Clinical Pharmacology,}

\section{School of Medicine,}

University College London,

5 University Street,

London WC1E 6JJ, UK

SIR-The recently published Report to the House of Lords of the Select Committee on Science and Technology entitled Civil Research and Development (HL 201 , HMSO) recommends that the "University Grants Committee selectivity exercise should be repeated in less than five years and the process be more open in future".

Not only is the openness important, but also the guarantee that like will be compared with like. This department, which was returned as Cost Centre 8 (other studies allied to medicine) was rated as average. It has research grants support of $£ 1,817,332$; has published 195 papers in the past four years; has pioneered medical imaging; has staff who are Royal Society Wellcome Foundation Gold Medallists and prize-winners in the BTG Academic Enterprise Competition; has spawned two companies; has one of the only two cyclotrons in the United Kingdom for medical imaging research; and has $15 \mathrm{PhD}$ students and $28 \mathrm{MSc}$ students. All this with an academic staff of five whole-time equivalent university posts and 40 graduate staff including National Health Service (NHS) and research grant staff.

I endeavoured to discover those departments that are above average and outstanding, so that we could learn how to improve our performance. According to The Times of 3 June 1986, the others graded in Cost Centre 8 (the London medical schools were excluded for reasons of space) were:

Outstanding: Aston; UMIST. Above average: King's. Average: Keele; Aberdeen; UWIST. Below average: Bradford; City; Exeter; Hull; Liverpool.

A short session perusing the Commonwealth Universities Year Book 1985 showed me that the only departments in these universities that might reasonably have been grouped into Cost Centre 8 were named as follows: ophthalmic optics (3); optometry (2): postgraduate medical (2); enviromental studies (2); nursing studies (2) communications and neurosciences (2); clinical communication (1); community health (1); health studies (1); physical education (1); medical sciences (1); biomedical engineering (1); medical physics (1).

If the UGC selectivity exercise really did compare these departments, then its absurdity is obvious, even to those unfamiliar with our interdisciplinary field which usually has active service commitments to the NHS as well. This is reinforced by the fact that those universities known to us personally as active in our field are not mentioned in The Times list, for example Surrey, Leeds, Sheffield.

I cannot accept the verdict of average for this department unless I can see openly that it has been done fairly. It is my belief that it may already have led to the loss of a medical-imaging research grant of $£ 470,400$ to the department.

The Select Committee reports, in addition, that it "...shares Sir Peter Swinnerton-Dyer's reluctance to establish within the UGC an elaborate assessment bureaucracy...". However, surely someone must be able to ensure that like is compared with like, and enable it to be seen to be operating fairly? Does it have to be elaborate?

Department of Bio-Medical

JOHN MALLARD

Physics \& Bio-Engineering,

University of Aberdeen and

Grampian Health Board,

Foresterhill,

Aberdeen AB9 2ZD, UK 